\title{
Review on (Yelow Pigment) Citrinin
}

\author{
Vasanthabharathi $\mathrm{V}^{*}$ and Jayalakshmi $\mathrm{S}$ \\ Faculty of Marine Sciences, S India \\ *Corresponding author: Vasanthabharathi V, Faculty of Marine Sciences, Parangipettai, Tamil Nadu, India
}

Submission: 㘹 October 10, 2018; Published: 些 November 26, 2018

Abstract

Many of the fungal species producing mycotoxin. Citrinin is produced by Penicillium, Aspergillus and Monascus species. It is a yellow pigmented mycotoxin and having antimicrobial, antioxidant activity. It is used in the production of rice wine preparation which is used as fish and meat preservative. Thus, the present review focused on Citrinin.

\section{Introduction}

The genus Penicillium contains approximately 100 toxigenic species, and the range of mycotoxin classes produced is much broader than that of any other genus [1]. listed 27 mycotoxins produced by 32 species which possessed varying range of toxicity. Penicillium toxins can be placed in two broad groups based on effect as renal and neurotoxins [2]. Mycotoxins are secondary metabolites produced by certain filamentous fungi. The toxic response due to fungal growth in food is called as mycotoxicosis. Aflatoxins are produced mainly by A. flavus, A. parasiticus and A. nominus. Aspergillus, Fusarium and Penicillium [3] and when ingested through food they intoxicate higher vertebrates including human. Monascus pigments, which are produced by various species of Monascus, often have been used as a natural colourant and as traditional natural food additives, especially in Southern China, Japan and Southeastern Asia. The limitation of wide using Monascus pigment is attributed to one of its secondary metabolites named citrinin.

Citrinin is a fungal metabolite was isolated from Penicillium citrinum in 1931. It was characterized as an antibiotic. Active against bacteria and also activity against bacteriophages, sarcomas, protozoa and animal cells. It is a quinine methide with two intra molecular hydrogen bonds. It is well established renal toxin affecting monogastric domestic animals such as pigs and dogs. Citrinin $\left(\mathrm{C}_{13} \mathrm{H}_{14} \mathrm{O}_{5}\right)$ is sparingly soluble in water but soluble in diluted sodium hydroxide, sodium carbonate, sodium acetate, methanol, acetonitrile, ethanol and most of other polar organic solvents [4]. It is a lemon-yellow crystalline substance, Melting point is $172{ }^{\circ} \mathrm{C}$ at room temperature. It possesses antibiotic, bacteriostatic, antifungal and antiprotozoal properties [5-8] reported occurrence of aflatoxin B1, citrinin and ochratoxin A in rice variety raised in five provinces of the central region of Vietnam. [9] explain about the Safety Evaluation of Citrinin in Foods. Anthraquinone-citrinin isolated from sea fan-derived P. citrinum PSU-F514 displayed moderate antibacterial activity against both $S$. aureus and methicillinresistant S. aureus [10].

\section{Application of citrinin}

There have been several reports on the isolation of bioactive metabolites, and the investigation of the ethyl acetate extracts from the broth and mycelia of $P$. citrinum has led to the extraction of nearly eighteen metabolites [11]. Bioactivity of citrinin possesses antibiotic, bacteriostatic, antifungal and antiprotozoal properties [12]. Citrinin was isolated from a Penicillium janczewskii strain found to inhibit the root-rot pathogen Heterobasidion annosum. Tarlov IM [13] carried out antimicrobial activity by using partially purified and purified citrinin. When partially purified citrinin was used it showed $8 \mathrm{~mm}$ zone of clearance against P. mirabilus, whereas $12 \mathrm{~mm}$ zone of clearance was observed against the same pathogen when purified citrinin was used. In the same research and Partially purified citrinin showed $4 \mathrm{~mm}$ zone of clearance against fungi P. thae. Purified citrinin showed $6 \mathrm{~mm}$ against $P$. thae. Another researcher [14] extracted citrinin from sponge associated Penicillium sp. and studied for its strong antibacterial activity against multi-drug resistant human pathogens like methicillin resistant $S$. aureus, rifampicin-resistant $S$. aureus, wild type $S$. aureus, vancomycinresistant E. faecium and also C. neoformans. Further, its having cytotoxicity against brine shrimp larvae.

Mazumde et al. [15] observed Minimum Inhibitory Concentration (MIC) against some gram positive strains viz. Staphylococcus aureus, Bacillus pumilus, Bacillus subtillis, Bacillus cereus, Klebsiella pneumoniae, Streptococcus pneumoniae, Lactobacillus arabinosus and gram negative strains E. Coli, Shigella dysenteriae, shigella sonnei, shigella boydii, Salmonella typhimurium, Proteus mirabilis and Vibrio cholerae by using citrinin. Antioxidant JBIR-124 [16], Cytotoxic penicitrinols C and E, topo II inhibitory tricitrinol $B$ and the cytotoxic dicitrinone B [17]. The isolation of five polyketides including two benzopyranones, one isochroman 
and two anthraquinone-citrinin derivatives were reported from the sea fan-derived fungus $P$. citrinum [18]. Citrinin used in the production of cheese and Sake. It plays a role in inducing motility of Paenibacillus polymyxa [17] Ciegler et al. [19] studied the biological activity of citrinin from Penicillium expansum. Pengnoi et al. [20] evaluate the effects of various purple rice varieties on the production of citrinin, and monacolin K, red pigments by Monascus purpureus CMU002U (UV-mutant strain) and their antioxidant properties.

\section{Characterization of citrinin}

Citrinin contaminated many food items has been widely reported. However, research on the citrinin biosynthesis pathway and its regulation mechanism in $P$. citrinum is rarely reported Iwahashi etal. [21] monitored the citrinin induced mRNA expression profiles in yeast using the ORF DNA microarray and Oligo DNA microarray. HPLC, TLC, enzyme linked immunosorbent assay, and bacteriostatic circle methods were used in the detection of citrinin [22]. A total of 19,967 uni genes were annotated by BLAST in Nr, Nt, Swiss-Prot and Kyoto Encyclopedia of Genes and Genomes (KEGG) databases. Transcriptome comparison between P. citrinum cultured with sucrose and glucose revealed 1085 differentially expressed unigenes. Among them, 610 were upregulated while 475 were downregulated under glucose as compared to sucrose [23]. Xu et al. [24] used TLC and HPLC to detect 40 strains of Monascus strains in yeast extract medium, and all the strains produced citrinin.

\section{Production of citrinin}

The fungi Penicillium citrinum was isolated from the sponge Callyspongia diffusa which was collected from Mandapam coast, Tamil Nadu, India. Optimization of citrinin was done by one factor at a time method. The optimum conditions for citrinin production were temperature $35{ }^{\circ} \mathrm{C}$, pH 7.0 salinty20ppt, carbon source-3\% glucose, Nitrogen source- $0.5 \%$ sodium nitrite, $3 \%$ rice bran Compared to commercially available carbon source (glucose), rice bran gave higher production of citrinin. About $1.50 \mu \mathrm{g} / \mathrm{ml}$ of citrinin was produced at optimized condition [12]. Likewise, another researcher Kaur et al. [25] carried out solid state fermentation for the production of pigments by using broken wheat (0.996 OD). The maximum pigment yield $(137.8 \mathrm{U} / \mathrm{g})$ was seen at $12^{\text {th }}$ day. Lai et al. [26] observed that Potato glucose medium is not conducive to citrinin production, however, yeast extract may promote the activity of citrinin synthase because of its rich nutrition and stimulate citrinin production. Li et al. [23] investigated the effect of different carbon sources on citrinin production by $P$. citrinum and used transcriptome analysis to study the underlying molecular mechanism. Results were indicated that glucose, used as the sole carbon source, could significantly promote citrinin production by $P$. citrinum in Czapek's broth medium compared with sucrose. Hasan [27] studied the citrinin accumulation under stress of camphor and blue-gum extract. Santos et al (2002) reported the effect of culture preservation techniques on patulin and citrinin production by Penicillium expansum. Wang et al. [28] observed the variability of citrinin production in Monascus type cultures. Prabhadevi et al. [29] isolated and characterized citrinin from P. chrysogenum [30].

\section{Conclusion}

Citrinin is a important mycotoxin, which is used in fermentation industry. It has antimicrobial activity against many pathogens hence it will be used as antibiotic in future.

\section{References}

1. Pitt JI, Leistner L (1991) Toxigenic Penicillium species. In: Smith JN, Hendersen RS (Eds.), Mycotoins and animal food. CRC press, Boca Raton, Florida, USA, pp. 39-55.

2. Pitt JI (1979) The Genus Penicillium and its Teleomorphic States Eupenicillium and Talaromyces, Academic Press, New York, USA, p. 629.

3. Sweeney MJ, Dobson ADW (1998) Mycotoxin production from Aspergillus, Fusarium and Penicillium species. Int J Food Microbiol 43(3): 141-158.

4. Deshpande SS (2002) Handbook of Food Toxicology. In: Marcel D (Ed.), Inc., New York, NY, USA, p. 424.

5. Hanika C, Carlton WW, Tuite J (1983) Citrinin mycotoxicosis in the rabbit. Food Chem Toxicol 21(4): 487-493.

6. Bilgrami KS, Sinha SP, Jeswal P (1988) Nephrotoxic and hepatoxic effects of citrinin in mice (Mus musculus). Proceed Ind Nation Sci Acad 54: 3537.

7. Berndt (1990) Ochratoxin-citrinin as nephrotoxins. In: Llewellyn GC, Rear PCO (Eds.), Biodeterioration Research Plenum Press, New York, USA, pp. 55-56.

8. Nguyen MT, Tozlovanu M, Tran TL, Pfohl Leszkowicz A (2007) Occurrence of aflatoxin B1, citrinin and ochratoxin $\mathrm{A}$ in rice in five provinces of the central region of Vietnam. Food Chemistry 105(1): 42-47.

9. Huiqin W, Shufen Z, Taifeng L, Dawei Z (2017) Introduction and safety evaluation of citrinin in foods. Journal of Food and Nutrition Sciences 5(5): 179-183.

10. Nanthaphong K, Vatcharin R, Chaveng P, Saowanit S, Souwalak P, et al. (2014) Acremonoside, a phenolic glucoside from the sea fan-derived fungus Acremonium polychromum PSUF125. Phytochemistry Letters 10: $50-54$.

11. Chen L, Liu W, Hu X, Huang K, Wu JL, et al. (2011) Citrinin derivatives from the marine-derived fungus Penicillium citrinum. Chem Pharm Bull 59(4): 515-517.

12. Abou Zeid AM (2012) Review on citrinin: Production, effect of some plant extracts and gene involved in its biosynthesis. Journal of Civil and Environmental Engineering 2: 113.

13. Vasanthabharathi V (2012) Bioactive substances of marine sponge associated microbes and their applications. pp. 191-196.

14. Subramani R, Kumar R, Prasad P, Albersberg W, Retheesh ST (2013) Cytotoxic and antibacterial substances against multi-drug resistant pathogens from marine sponge symbiont: Citrinin a secondary metabolite of Penicillium sp. Asian Pac J Trop Biomed 3(4): 291-296.

15. Mazumder PM, Mazumder R, Mazumder A, Sasmal DS (2002) Antimicrobial activity of the mycotoxin citrinin obtained from the fungus Penicillium citrinum. Anc Sci Life 21(3): 191-197.

16. Kawahara T, Takagi M, Shinya K (2012) JBIR-137 and JBIR-138, new secondary metabolites from Aspergillus sp. FA75. J of antibiotic 65: 535538.

17. Khamthong N, Rukachaisirikul V, Phongpaichit S, Preedanon S, Sakayaroj J (2012) Bioactive polyketides from the sea fan-derived fungus Penicillium citrinum PSUF51. Tetrahedron 68(39): 8245-8250.

18. Park SY, Kim R, Ryu CM, Choi SK, Lee CH, et al. (2008) Citrinin, a mycotoxin from Penicillium citrinum, plays a role in inducing motility of Paenibacillus polymyxa. FEMS Microbiol Ecol 65(2): 229-237. 
19. Ciegler A, Vesonder RF, Jackson LK (1977) Production and biological activity of patulin and citrinin from Penicillium expansum. Appl Environ Microbiol 33(4): 1004-1006.

20. Pengnoi P, Mahawan R, Khanongnuch C, Lumyong S (2017) Antioxidant properties and production of monacolin $\mathrm{k}$, citrinin, and red pigments during solid state fermentation of purple rice (Oryzae sativa) varieties by Monascus purpureus. Czech J Food Sci 35(1): 32-39.

21. Iwahashi H, Kitagawa E, Suzuki Y, Ueda Y, Ishizawa YH, et al. (2007) Evaluation of toxicity of the mycotoxin citrinin using yeast ORF DNA microarray and Oligo DNA microarray. BMC Genomics 8: 95.

22. Chen Y, Xu GR, Yu HL (2004) Citrinin analysis using HPLC. Food and Fermentation Industries 30(1): 118-123.

23. Li,T, Jiang G, Hongxia Qu, Wang Y, Xiong Y, et al. (2017) Toxins 9(69): 2-18.

24. Xu GR, Chen Y, Gu YM (2009) Screening of monascus strains with low content citrinin production ability. Journal of Wuxi University of Light Industry 19: 58-61.
25. Kaur S, Arora N, Kaur S (2017) Characterization of yellow pigments produced by Pencillium sp. under solid state cultivation. J Biotechnol Biomater 7: 259.

26. Lai WH, Xu Y, Li YP (2004) Study on factors of producing citrinin by Monascus spp. China Brewing 2: 12-14.

27. Hasan HAH (1993) Citrinin accumulation under stress of camphor and blue-gum extract. J Islam Acad Sci 6(1): 29-32.

28. Wang YZ, Ju XL, Zhou YG (2005) The variability of citrinin production in Monascus type cultures. Food Microbiol 22(1): 145-148.

29. Prabhadevi, DeSouza L, Kamat T, Rodrigues C, Naik CG (2009) Batch culture fermentation of Penicillium chrysogenum and a report on the isolation, purification, identification and antibiotic activity of citrinin. Ind J Mar Sci 38(1): 38-44.

30. Blanc PJ, Laussac J, Le Bars P, Le Bars MO, Loret A, et al. (1995). Characterization of Monascidin A from Monascus as citrinin. Int J Food Microbiol 27(2-3): 201-213.
Creative Commons Attribution 4.0 International License

For possible submissions Click Here
Submit Article

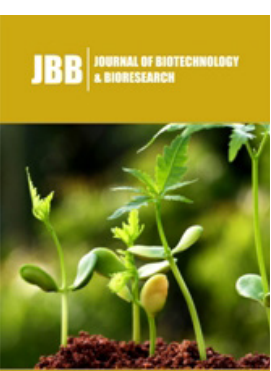

Journal of Biotechnology \& Bioresearch

\section{Benefits of Publishing with us}

- High-level peer review and editorial services

- Freely accessible online immediately upon publication

- Authors retain the copyright to their work

- Licensing it under a Creative Commons license

- Visibility through different online platforms 